\title{
IN-SITU X-RAY REFLECTIVITY STUDY ON GROWN DYNAMICS OF SPUTTER DEPOSITED GOLD ON SLICON*
}

\author{
R. P. Chiarello, H. K. Kim, T. Roberts, D. J. Miller, R. T. Kampwirth, \\ K. E. Gray, and H. You \\ Materials Science Division \\ Argonne National Laboratory \\ Argonne, Il 60439
}

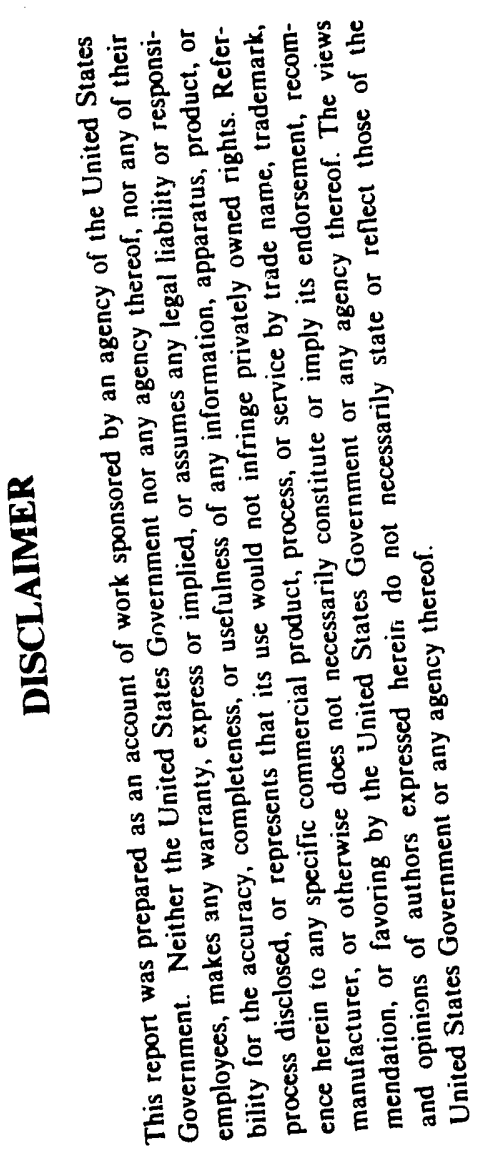

The submitted manuscript has been authored by a contractor of the U. S. Government under contract No. W-31-109-ENG-38.

Accordingly, the U.S. Government retains a nonexclusive, royalty-free license to publish or reproduce the published form of this contribution, or allow others to do so, for U.S. Government purposes.

NOVEMBER 1991

Submitted to MRS Meeting, Boston, MA, December 2-6, 1991.

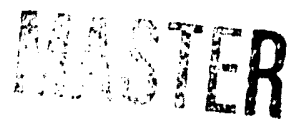

*Work Supported by the U.S. Department of Energy, BES-Materials Sciences, under Contract \#W-31-109-ENG-38. 
B.P.CHIARELLQ， H.K.KIM， T.ROBERTS， D.J.MILLER， R.T.KAMPWIRTH， K'.E.GRAY, AND H.YOU

Argonne National Laboratory, MSD, Argonne, Il 60439

\section{ABSTRACT}

We performed in-situ x-ray reflectivity measurements to study the growth dynamics of gold sputter deposited onto silicon, using an $x$-ray scattering chamber equipped with a faced magnetron source where the substrate is held at a right angle relative to the sputtering guns. By operating the guns at low power ( 1 watt) and under 20 mTorr $A r$, we could control the gold deposition rate to less than $1 \dot{A} / s e c$. The observed $\bar{x}$-ray reflectivity for gold deposited onto a silicon substrate held at room temperature may be consistent with nucleated island growth for average gold particle sizes less than $50 \dot{A}$. Above $50 \dot{A}$, the reflectivity data indicates that the gold film uniformly covers the silicon surface, and that as the film thickness is increased the gold-vacuum interface gets progressively rougher. Detailed analysis of room temperature data is in progress, as is the temperature dependence on the roughness of the gold vacuum interface.

\section{INTRODUCTION}

The growth dynamics of thin solid films is sensitively dependent upon the deposition method and conditions. Thin solid film formation by particle deposition under nonequilibrium conditions has been an active area of theoretical and computer simulation research for over ten years[1]. In spite of this, there has been only a limited number of experimental works done. The synchrotron $x$-ray reflectivity technique is a sensitive structural probe on microscopic length scales.Here we report preliminary results of the dependence of growth conditions on sputter deposited gold films on silicon studied by in-situ $x$-ray reflectivity.

\section{EXPERIMENTAL}

Synchrotron $x$-ray reflectivity measucements were performed on beamline $\mathrm{X} 6 \mathrm{~B}$, at the National Synchrotron Light Source, Brookhaven National Laboratory. The wavelength of the incident $x$-rays was $1.55 \dot{A}$. Two pairs of tantalum slits were used to define the unfocused incoming beam to $2 \mathrm{~mm}$ by $2 \mathrm{~mm}$. An $x$-ray compatible sputtering chamber, whose details are presented elsewhere[2], was used with a faced magnetron sputtering system. The chamber was mounted on a Huber four circle diffractometer and had a base pressure of $5 \times 10^{-5}$ torr. Research grade argon gas was used for sputtering, and an Ortec high voltage power supply, tunable from 
0-3000 volts at 0-20 mA, powered the sputtering guns. The deposition rate was $0.2-1$ A $/$ sec for data presented here. The reflectivity scans were recorded after sputtering for a fixed time. Silicon samples were ultrasonically cleaned for five minutes each in acetone, methyl alcohol, and deionized water. They were then baked in vacuum to $475 \mathrm{~K}$ in order to remove surface contaminants such as hydrocarbons and water.

\section{RESULTS}

The $x$-ray reflectivity geometry is shown in figure 1 for $x-$ rays incident upon a film having index of refraction $n_{1}$, which was grown on a semi-infinite substrate with index of refraction $n_{2}$. The reflection amplitude for an ideally sharp interface can be written down as [3]

$A=\frac{r_{01}+r_{12} e^{-i Q D}}{1+r_{01} r_{12} e^{-i Q D}}$

where $r_{i j}$ is the Fresnel reflection amplitude of classical optics, and $L$ the layer thickness. The deviation of the data from this equation is a sensitive measure of interfacial roughness.

Figure $2(a)$ and (b) show the reflectivity data as a function of momentum transfer recorded at $300 \mathrm{~K}, 10 \mathrm{mTorr}$ Ar gas pressure and $400 \mathrm{~K}, 10 \mathrm{mTorr}$ Ar gas pressure, respectively. In Eigure 2(a), the top most reflectivity was recorded after sputtering for two minutes. Below the critical momentum transfer $\left(Q_{c}=(4 \pi / \lambda) \mathrm{sin} \theta_{c}\right.$ ) incident $x$-rays experience total external reflection. The values $\left(Q_{c}\right)$ for gold and silicon are 0.080 and $0.032 \dot{A}^{-1}$ respectively. The two minute data clearly shows the silicon critical angle, which may be consistent with gold islands partially covering the silicon up to gold particle sizes of $50 \dot{A}$. The oscillations in the data are due to interference between the silicon-gold and gold-vacuum interfaces and the frequency of oscillation is proportional to film thickness. The roughness of the gold-vacuum interface is manifested by a decrease in the reflected intensity above $Q_{c}$. The data suggests that the gold surface roughness increases with increasing deposition time.

In the early growth stages, up to approximately $100 \dot{A}$, the $400 \mathrm{~K}$ data is very similar to the $300 \mathrm{~K}$ data, but the data exhibit marked differences thereafter. For example, the oscillation frequency of the $300 \mathrm{~K}$ data uniformly increases up to 60 minutes of deposition, indicating that a film with a relatively smooth surface is growing. However, the $400 \mathrm{~K}$ film oscillation frequency does not uniformly increase after 14 minutes of deposition. speculatively, this is suggestive of a wetting transition, where the $300 \mathrm{~K}$ gold film is growing uniformly thick, but the $400 \mathrm{~K}$ gold may be growing in bulk-like droplets. 
Figure 3 shows powder diffraction scans of four gold/silicon samples measured using copper $K_{\alpha 1}$ radiation from an in-house rotating anode source. The films are approximately $3000 \dot{A}$ thick, and were sputtered, from top to Dottom, at 1 mTorr Ar pressure, $300 \mathrm{~K}$, 1 mTorr, $220 \mathrm{~K}, 10 \mathrm{mTorr}, 300 \mathrm{~K}$, and $10 \mathrm{mTorr}, 220 \mathrm{~K}$. From figure 3 , and corresponding rocking curve scans, it was determined that the films made at 1 mTorr are two dimensional(2D) powders made up of randomly oriented crystallites with (111) faces parallel to the substrate surface. The films made at $10 \mathrm{mTorr}$ are also $2 \mathrm{D}$ powders, but the crystallites have a distribution of(111) and (200) faces oriented parallel to the substrate surface. Data recorded at $400 \mathrm{~K}$, 10 mTorr is consistent with the low temperature 10 mTorr results.

\section{SUMMARY}

We have exhibited the practicality of performing in-situ $x-$ ray reflectivity studies for sputter deposition. Preliminary results indicate that film crystallite orientation, and growt.h mode are sensitive to deposition conditions. Significantly, the surface roughness of a growing film increases with time or, equivalently, with film thickness. This result is independent of the variation of deposition conditions studied here. An investigation of the dynamic scaling behavior of growing thin solid films under a variety of sputter deposition conditions is in progress.

\section{ACKNOWLEDGEMENTS}

This work supported by U.S. Department of Energy, 'Division of Basic Energy Sciences.

\section{CITATIONS}

1. F. Family, Physica A 168, 561 (1990)

2. J.Q. Zheng, M.C. Shih, X.K. Wang, S. Williams, P. Dutta, R.P.H. CHang, and J. B. Ketterson, J. Vac. Sci. Tehonol. A 2 (1), 128 (1991)

3. M. Born and E. Wolf, Principles of Optics, (Pergamon Press, Oxford, 1970) 


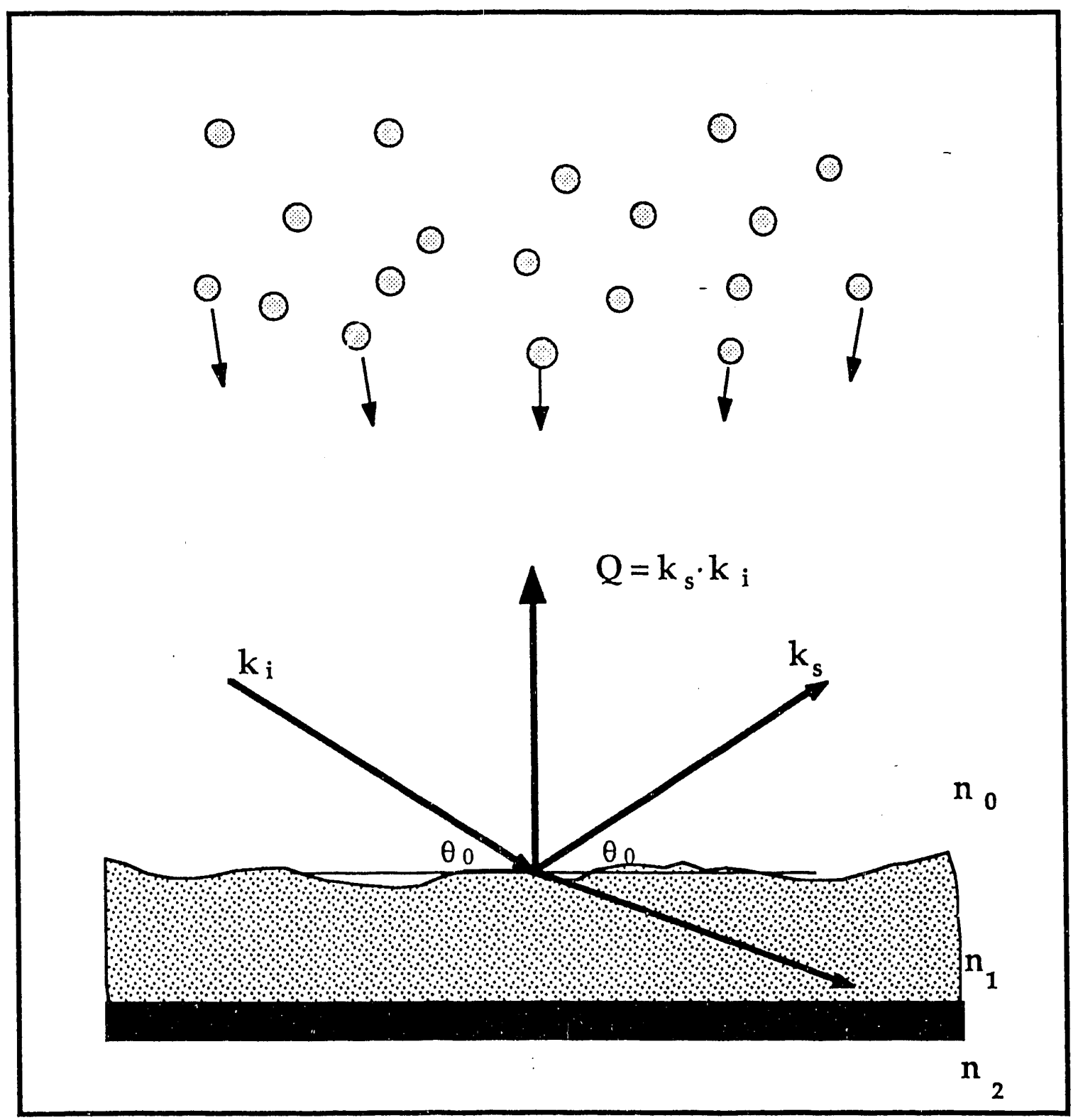

Figure 1: In-situ specular $x$-ray reflectivity geometry for $x$-rays incident upon a sputter deposited film with complex index of refraction $n_{1}$. 


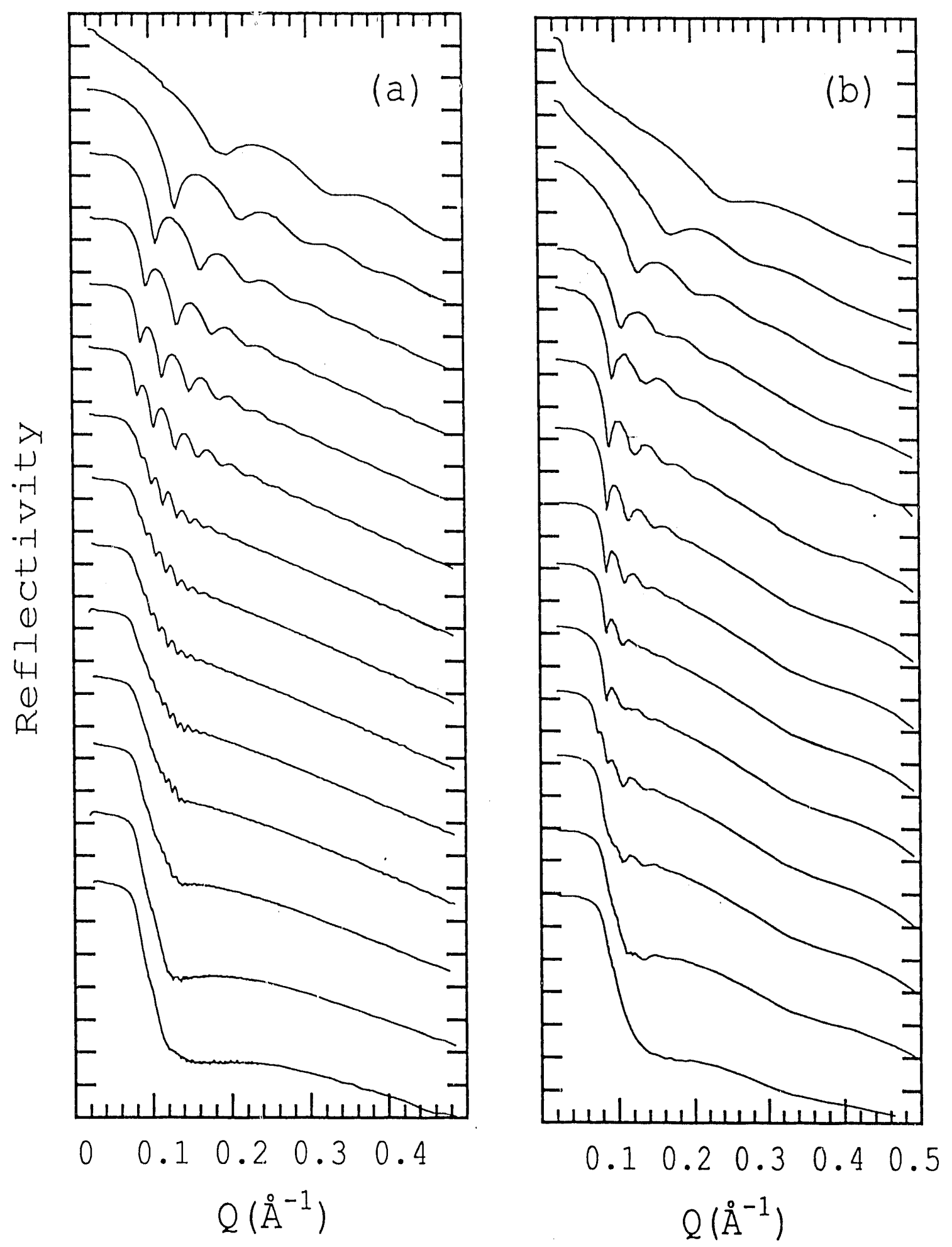

Figure 2: Reflectivity versus $Q$ for gold sputter deposited on silicon. (a) The substrate temperature was $300 \mathrm{~K}$ and the deposition times from top to bottom were $2,4,6,8,10$, $12,20,24,28,36,44,60,90$, and 120 minutes. (b) Substrate temperature was $400 \mathrm{~K}$ and the deposition times were, from top to hottom: 1, 2, 4: 6: 8, 10, 12, 14, 16: $18,28,40,70$, and 130 minutes. 


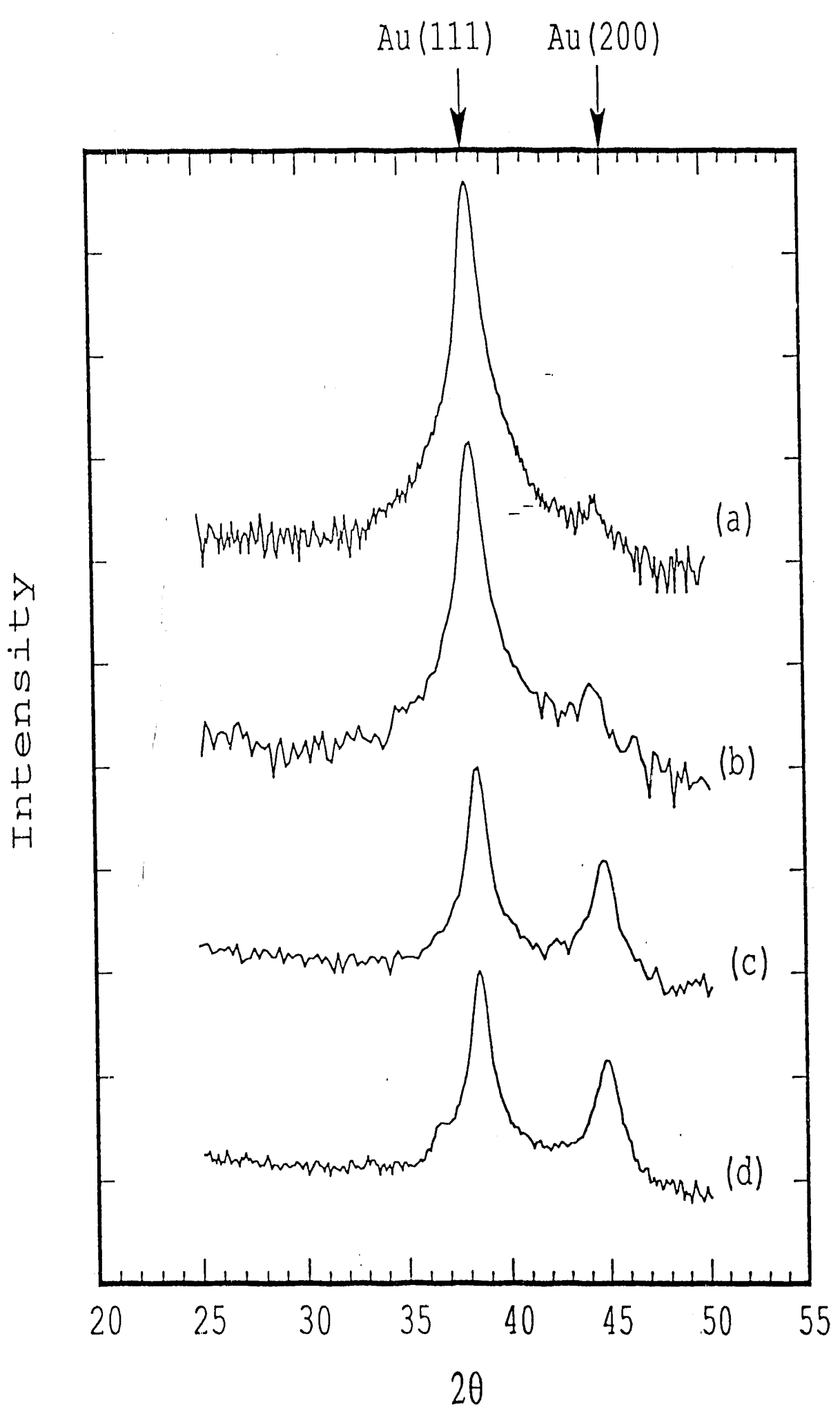

Figure 3: Powder diffraction of gold films sputtered deposited on silicon to a thickness of approximately $3000 \dot{A}$. The deposition conditions were; (a) $220 \mathrm{~K}$ and 1 mTorr Ar pressure, (b) $300 \mathrm{~K}$ and $1 \mathrm{mTorr}$ Ar pressure, (c) $230 \mathrm{~K}$ and 10 mTorr Ar pressure, and (d) $300 \mathrm{~K}$ and $10 \mathrm{mTorr} A r$ pressure. 

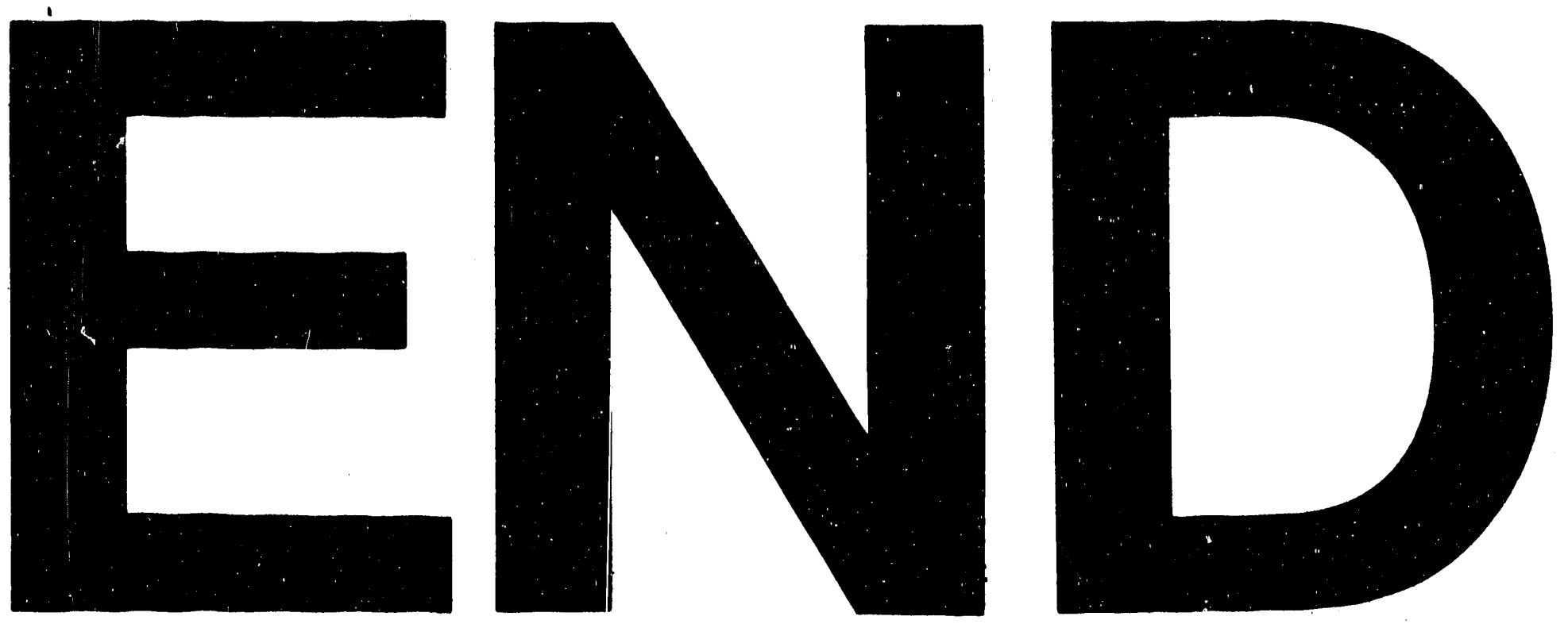

1
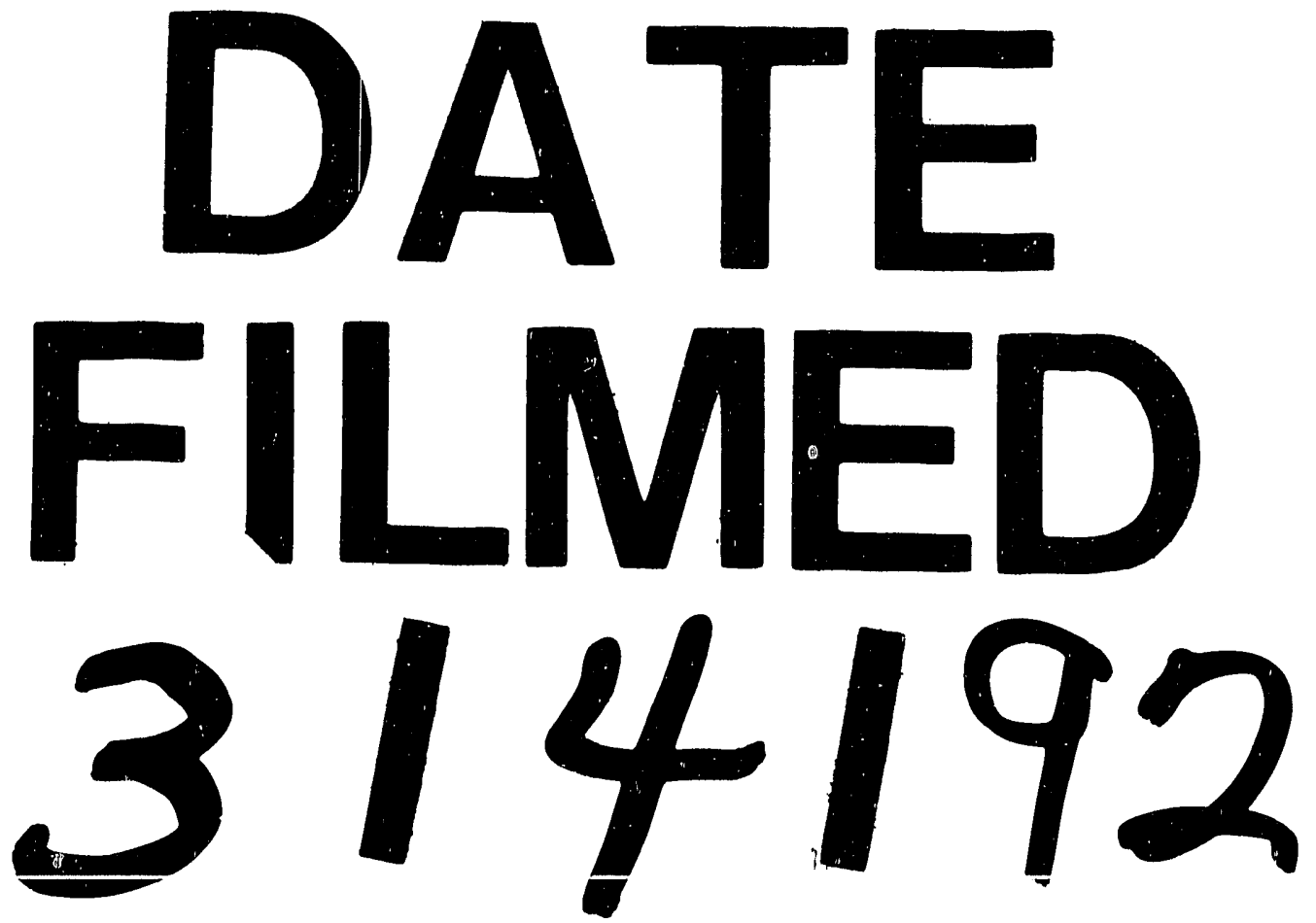
